\title{
Regulation of testicular tight junctions by gonadotrophins in the adult Djungarian hamster in vivo
}

\author{
Gerard A Tarulli ${ }^{1,2}$, Sarah J Meachem ${ }^{1,2}$, Stefan Schlatt ${ }^{3}$ and Peter G Stanton ${ }^{1}$ \\ ${ }^{1}$ Prince Henry's Institute of Medical Research, PO Box 5152, Clayton, Victoria 3168, Australia, ${ }^{2}$ Department of \\ Anatomy and Developmental Biology, Monash University, Clayton, Victoria 3168, Australia and ${ }^{3}$ Department of Cell \\ Biology and Physiology, University of Pittsburgh, Pittsburgh, Pennsylvania 15261, USA
}

Correspondence should be addressed to P G Stanton; Email: peter.stanton@princehenrys.org

\begin{abstract}
This study aimed to assess the effect of gonadotrophin suppression and FSH replacement on testicular tight junction dynamics and blood-testis barrier (BTB) organisation in vivo, utilising the seasonal breeding Djungarian hamster. Confocal immunohistology was used to assess the cellular organisation of tight junction proteins and real-time PCR to quantify tight junction mRNA. The effect of tight junction protein organisation on the BTB permeability was also investigated using a biotin-linked tracer. Tight junction protein (claudin-3, junctional adhesion molecule (JAM)-A and occludin) localisation was present but disorganised after gonadotrophin suppression, while mRNA levels (claudin-11, claudin-3 and occludin) were significantly (two- to threefold) increased. By contrast, both protein localisation and mRNA levels for the adaptor protein zona occludens-1 decreased after gonadotrophin suppression. FSH replacement induced a rapid reorganisation of tight junction protein localisation. The functionality of the BTB (as inferred by biotin tracer permeation) was found to be strongly associated with the organisation and localisation of claudin-11. Surprisingly, JAM-A was also recognised on spermatogonia, suggesting an additional novel role for this protein in trans-epithelial migration of germ cells across the BTB. It is concluded that gonadotrophin regulation of tight junction proteins forming the BTB occurs primarily at the level of protein organisation and not gene transcription in this species, and that immunolocalisation of the organised tight junction protein claudin-11 correlates with BTB functionality.

Reproduction (2008) 135 867-877
\end{abstract}

\section{Introduction}

During puberty in higher mammals, Sertoli cells undergo a complex process of structural and biochemical maturation to become differentiated epithelial cells in readiness to support germ cell (GC) development. This process involves the creation of the blood-testis barrier (BTB), formed largely by the binding of transmembrane tight junction (TJ) proteins on adjacent Sertoli cells (reviewed in Setchell et al. 1988, 2003). This barrier resides near the basement membrane of the seminiferous tubules and divides the seminiferous epithelium into basal and adluminal compartments. The BTB allows for the establishment of a specialised epithelial microenvironment, separated from factors found in the interstitium, which is conducive to meiotic and post-meiotic GC development. In the absence of a functional BTB, spermatogenesis continues only as far as spermatocytes in rodents (Cavicchia \& Sacerdote 1991, Gow et al. 1999, Morales et al. 2007), highlighting the indispensability of the BTB. While intensively studied (for reviews see (Lui et al. 2003, Turner 2006, Matter \& Balda 2007) the regulation of TJ proteins in the testis is poorly understood. The present study investigates the regulation of TJ proteins by gonadotrophins in a model of natural gonadotrophin suppression as induced by a short photoperiod - the seasonal-breeding Djungarian hamster.

Sertoli cell TJs contain three well-defined transmembrane protein groups, the claudins, particularly claudin-11 (also known as oligodendrocyte transmembrane/specific protein; Morita et al. 1999) and claudin-3 (Meng et al. 2005), occludin (Furuse et al. 1993, Morita et al. 1999) and the junctional adhesion molecule (JAM) family of proteins (Martin-Padura et al. 1998, Bazzoni etal. 2000, Xia et al. 2005) that are bound to intracellular plaque proteins such as zona occludens family members (ZO-1 and ZO-2; Stevenson et al. 1986, Fanning et al. 1998), which tether TJ proteins to the underlying actin cytoskeleton (Fanning et al. 1998). In the rodent testis, TJ proteins are found in close spatial association with the basal ectoplasmic specialisation, which is a testis-specific actinbased adherens junction containing the actin-bundling protein espin (Bartles et al. 1996, Lee \& Cheng 2004), involved in Sertoli-Sertoli and Sertoli-GC adhesion. Claudin and occludin TJ proteins are thought to be classical barrier-forming proteins, while JAM family members have also been associated with cellular adhesion (Morris et al. 2006), trans-epithelial cellular migration (Woodfin et al. 2007) and cellular polarity (Rehder et al. 
2006) in other mammalian tissues, with recent evidence suggesting that they may also play a role in spermatogenesis in rodents (Zen et al. 2005, Wang \& Cheng 2007). JAM family members (JAM-B and JAM-C) have been identified on both Sertoli and GCs in rodents, while claudins and occludin have been localised exclusively to inter-Sertoli cell TJs in this species (Wang \& Cheng 2007). To date, JAM$A$ has been identified at inter-Sertoli cell junctions and the tails of elongated spermatids within the epididymis of rodents (Shao et al. 2008).

Various studies suggest that gonadotrophins play an important role in testicular TJs. Both follicle-stimulating hormone ( $\mathrm{FSH}$ ) and testosterone increase the function of rat Sertoli cell TJs in vitro (Janecki et al. 1991, Kaitu'u-Lino et al. 2007) in part by up-regulating claudin-11 mRNA and protein (Kaitu'u-Lino et al. 2007), although FSH has also been found to down-regulate claudin-11 mRNA expression in cultured mouse Sertoli cells (Hellani et al. 2000). In vivo, selective knockout of the Sertoli cell androgen receptor in mice results in a limited reduction in claudin-11 protein (Tan et al. 2005 2005), but a substantial tenfold decrease in claudin-3 gene transcription (Meng et al. 2005). In the Djungarian hamster testis, claudin-11 protein persists after gonadotrophin suppression, but its localisation is disorganised (Tarulli et al. 2006). Although a number of other factors have been shown to regulate TJs, including cytokines (interferon- $\gamma$, tumour necrosis factor- $\alpha$, transforming growth factor- $\beta 3$ (Lui et al. 2001, Li et al. 2006)), intracellular $\mathrm{Ca}^{2+}$ concentration (Grima et al. 1998) and protein kinase activities (Li et al. 2001), the mechanism(s) by which gonadotrophins regulate TJ gene expression, localisation and BTB function in vivo remain unclear.

We used the Djungarian hamster to assess the effect of gonadotrophin suppression and FSH replacement on TJ dynamics in vivo. This naturally occurring animal model provides an opportunity to study TJ breakdown and reformation, as exposure to short-day length $(\mathrm{SD}, 8 \mathrm{~h}$ light:16 $\mathrm{h}$ darkness) alone suppresses pituitary FSH and luteinising hormone (LH), and consequently testicular testosterone. This results in a disruption of spermatogenesis primarily at the level of spermatogonial development (Bergmann 1987, Lerchl et al. 1993, Schlatt et al. 1995, Meachem et al. 2005, 2007), and also disrupts the function of the BTB (Bergmann 1987). Low numbers of primary spermatocytes remain within the epithelium of SD hamsters (Bergmann 1987); however, no pachytene spermatocytes are observed (Meachem et al. 2005). Recrudescence in Djungarian hamsters occurs following the restoration to LD photoperiod (LD, $16 \mathrm{~h}$ light: $8 \mathrm{~h}$ darkness), resulting in the normalisation of serum gonadotrophins ( $\mathrm{FSH}$ at 3 days and $\mathrm{LH}$ at 21 days; Furuta et al. 1994), BTB function, (Bergmann 1987) and restoration of spermatogenesis (Lerchl et al. 1993). However, $\mathrm{LH} /$ androgen involvement in recrudescence appears less important as GC numbers are not different between SD animals treated with $\mathrm{FSH}$ alone or when FSH is co-administered with the androgen receptor antagonist, flutamide (Meachem et al. 2005).

Both sperm production (Lerchl et al. 1993) and fertility (Niklowitz et al. 1997) in the Djungarian hamster are highly dependent on $\mathrm{FSH}$, with testosterone known to be necessary only for mounting behaviour (Lerchl et al. 1993). Despite the changes in the TJ functionality, relatively little is known about the regulation of $\mathrm{TJ}$ and associated proteins in this species. We therefore hypothesised that after exposure to SD conditions, Sertoli cell TJ protein localisation will be disorganised and mRNA levels decreased, and that FSH replacement to SD animals will reverse this profile. The aims of this study were to establish the effect of gonadotrophin suppression and FSH replacement on TJ protein organisation and mRNA levels, as well as to determine the relationship between $\mathrm{TJ}$ organisation and BTB functionality.

\section{Results \\ Stage-dependency of tight junction localisation and BTB functionality over the cycle of the seminiferous epithelium}

In the adult rodent, there are indications for stagedependency of TJ protein localisation (Xia et al. 2005, Tarulli et al. 2006), but this has not been assessed in detail. To further investigate this stage-dependency, we assessed the expression and localisation of the major tight junction protein, claudin-11, over the cycle of the seminiferous epithelium in the LD hamster. This protein was selected as the claudin-11 knockout mouse is infertile, with an absence of tight junction fibrils in Sertoli cells (Gow et al. 1999). Representative images of the change in claudin-11 localisation (pale blue) over the cycle of the seminiferous epithelium are shown in Fig. 1A. Claudin-11 localisation was predominantly basal relative to GCs residing on the basement membrane at stages I-III and XI-XII, while adluminal relative to GCs residing on the basement membrane at stages VII-VIII. An intermediate phenotype was observed at stages IV-VI and IX-X (Fig. 1A).

To qualitatively assess the relationship between BTB functionality and claudin-11 localisation, a biotin-NHS conjugated solution was injected into freshly dissected testes before fixation (Meng et al. 2005). In the adult LD hamster testis (Fig. 1B), a strong biotin staining was observed in the interstitium. The degree of biotin permeation into the epithelium exhibited stage-dependency, with a similar pattern as claudin-11 reactivity. In Fig. 1B, two tubules are seen which have been staged and labelled based on their acrosomal morphologies as revealed by PNA reactivity (red). In tubules of stages VII-VIII, extensive claudin-11 reactivity can be seen above GCs residing along the basement membrane. Biotin permeated the epithelium only as far as this organised claudin-11 reactivity. In the adjacent tubule (stage 

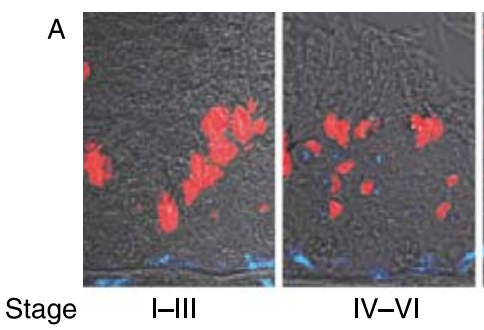

IV-VI

B

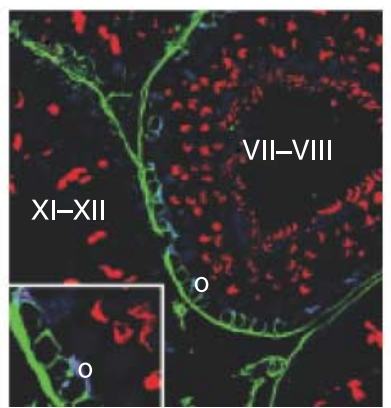

grouping XI-XII), only focal areas of biotin permeation was detectable around basal GCs, while claudin-11 expression was either absent or present at low levels along the basement membrane. At all stages in the LD animal, biotin permeated the seminiferous epithelium only as far as organised claudin-11 immunoreactivity.

\section{Quantitation of stage-dependency of tight junction localisation over the cycle of the seminiferous epithelium}

The frequency of claudin-11 localisation in staged tubules was then assessed, and expressed as \% basal (Fig. 2A), \% intermediate (Fig. 2B) and \% adluminal (Fig. 2C) relative to GCs residing on the basement membrane. In stages I-III, localisation of claudin-11 was predominantly at the basement membrane $(72 \%$ basal, $9 \%$ intermediate, $0 \%$ apical relative to GCs residing on the basement membrane; Fig. 2). Localisation was intermediate in stages IV-VI $(22 \%$ basal, 63\% intermediate and 4\% apical; Fig. 2) and predominantly adluminal relative to GCs residing on the basement membrane during stages VII-VIII (0\% basal, $29 \%$ intermediate and $71 \%$ apical; Fig. 2). Localisation returned to a more basal phenotype at stages IX-XII.

The intensity of claudin- 11 immunoreactivity was highest in stages VII-VIII (91\%) and IX-X (82\%) and lowest in stages I-III (42\%) and XI-XII (48\%; Fig. 2D, filled columns). Similar relative frequencies were observed in the assessment of biotin infiltration with the highest occurring during stages VII-VIII (92\%) and lowest during stages I-III (28\%) and XI-XII (22\%; Fig. 2D, open columns).

\section{Functionality of the BTB after natural gonadotrophin suppression and exogenous FSH-replacement}

The organisation of claudin-11 in the Djungarian hamster has been assessed previously (Tarulli et al. 2006), with

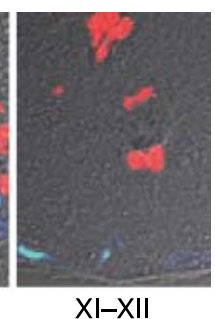

Figure 1 Localisation and function of the blood-testis barrier is stage-dependent. (A) Confocal immunohistology of claudin-11 (pale blue) localisation in tubule stage groupings based on PNA-lectin reactivity (red). (B) Testis section from adult LD (active spermatogenesis) labelled with claudin-11 (pale blue) and PNA-lectin 546 (red) to allow for staging. The testes were injected with an NHS-biotin tracer to assess the permeability of the blood-testis barrier (biotin, green). The interface between biotin and claudin-11 staining is indicated by an open circle. Insert is an enlarged portion of the stages VII-VIII epithelium illustrated in this panel.

disorganisation of claudin-11 protein observed after gonadotrophin suppression, and a rapid reorganisation after exogenous FSH replacement. To correlate claudin-11 localisation with BTB functionality, in this study we assessed biotin permeation in these states. In the SD hamster (gonadotrophin suppressed; Fig. 3A), where the BTB is known to be non-fpunctional (Bergmann 1987), biotin (green) was able to permeate the entire seminiferous epithelium. FSH treatment to SD hamsters resulted in the exclusion of biotin tracer from the centre of tubules after 10 days (Fig. 3C) but not 2 days (Fig. 3B). In all panels, biotin permeated the seminiferous epithelium only as far as organised claudin-11 reactivity (Fig. 3A-C).
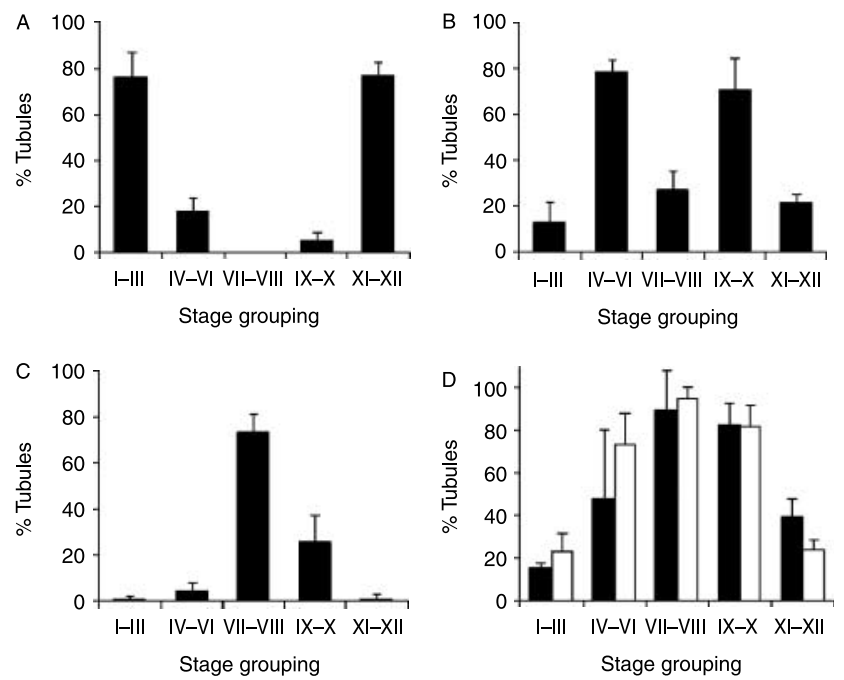

Figure 2 Organisation and expression of tight junction protein claudin-11 is stage-dependent. The percentage of stage-grouped seminiferous tubules exhibiting basal (A), intermediate (B) or adluminal (C) claudin-11 expression, relative to germ cells residing on the basement membrane. (D) The percentage of stage-grouped seminiferous tubules exhibiting biotin infiltration $(\square)$ or claudin-11 expression $(\boldsymbol{\square})$. Error bars signify s.D. $(n=5)$. 

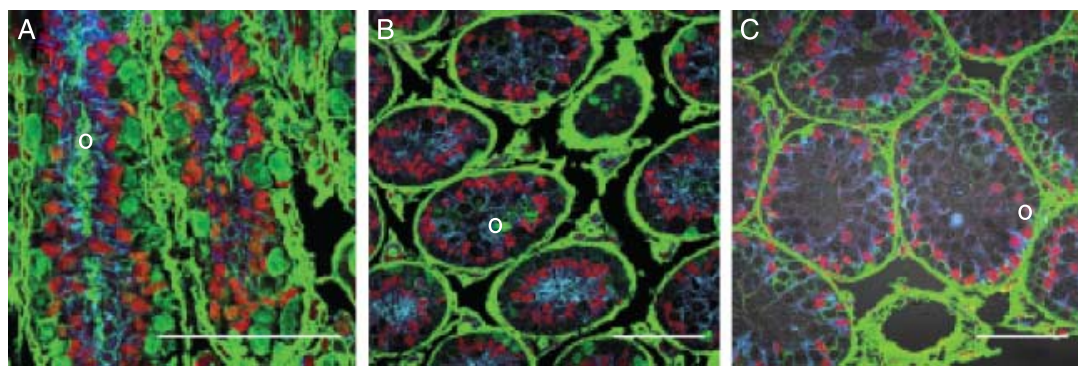

Figure 3 FSH replacement restores the blood-testis barrier functionality after natural gonadotrophin suppression. Confocal immunohistology of claudin-11 (pale blue), GATA4 (marker of all somatic cells, red) and biotin (green) in adult SD (repressed spermatogenesis, A), and adult SD hamster treated with $\mathrm{FSH}$ for 2 days (B) or 10 days (C). The interface between biotin and claudin-11 staining is indicated by open circles. See the text for description of localisation (bar $=50 \mu \mathrm{m})$.

\section{FSH regulates claudin-3 and espin localisation}

We then assessed the localisation of the other key tight junction proteins in this hamster model. Lowlevel claudin-3 reactivity was observed in the interstitium of immature (d5) hamsters that lack tight junctions (Fig. 4A, open square), while a disorganised espin reactivity was detectable in the seminiferous epithelium (open triangle). A strong claudin-3 reactivity was observed in blood vessels of the adult LD, SD and $\mathrm{FSH}$-treated hamster (B-F, open squares). In the LD animal (Fig. 4B), claudin-3 reactivity was detectable only in association with elongated spermatids (open circle) in the seminiferous epithelium. Espin, a testis-specific adherens junction marker, was used as a standard marker for localisation and reactivity in this figure and also in the subsequent figures. Espin localisation was found around cells adjacent to the basement membrane and in association with elongating spermatids (Fig. 4B, open triangles). In the SD hamster (Fig. 4C), claudin-3 reactivity was present in the apical regions of Sertoli cells (open circle), while a low-level espin reactivity was observed in the apical Sertoli cell cytoplasm and around GCs (open triangle). After 2 days of FSH treatment (Fig. 4D) intense claudin-3 reactivity was present at the basal aspect of Sertoli cells localised around the GCs and basement membrane in this region, and also at the newly forming lumen (open circles). Espin localised at the basal aspect of Sertoli cells, above and around GCs residing on the basement membrane. Espin expression co-localised with claudin-3 in many regions and also extended through much of the epithelium (Fig. 4D, open triangles). After 4 days of $\mathrm{FSH}$ treatment (Fig. 4E), claudin-3 continued to be expressed adjacent to GCs residing along the basement membrane (open circle); however, the reactivity was reduced at luminal regions, while espin reactivity persisted at luminal regions (open triangle). After 10 days of FSH treatment (Fig. 4F), claudin-3 expression was observed in some tubules (open circle), but absent in those exhibiting more advanced GCs. At this time point, espin continued to organise above GCs residing along the basement membrane and through much of the epithelium (Fig. 4F, open triangle).

\section{FSH regulates JAM-A localisation}

In all groups, intense JAM-A reactivity was observed in interstitial blood vessels in the immature (d5; Fig. 5A, open square) and adult (Fig. 5C-F, open squares) hamster.
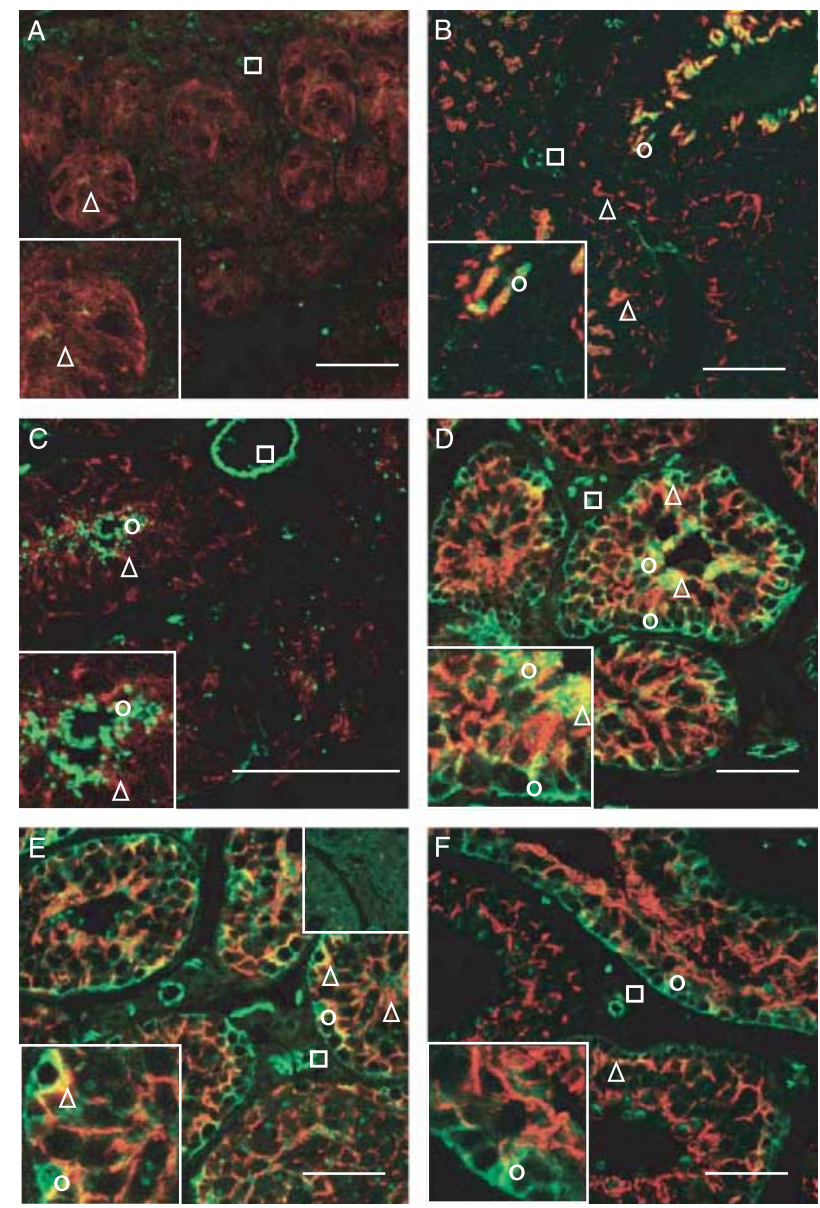

Figure $4 \mathrm{FSH}$ regulates claudin-3 and espin localisation. The localisation of tight junction protein claudin-3 (green, open circles) and the testis-specific adherens junction protein espin (red, open triangles) by confocal immunofluorescence, in testes from the immature (day 5) hamster (A) and adult long-day (LD) (B), short-day (SD) (C) and SD hamster treated with $\mathrm{FSH}$ for 2, 4 and 10 days (D, E and $\mathrm{F}$ respectively). See the text for description of localisation. Lower inserts are enlarged regions of the same micrograph. The upper insert in $\mathrm{E}$ is a control where the primary antibody was substituted with an equivalent concentration of a non-specific antibody from the same species. Open squares indicate claudin-3 reactivity in blood vessels (bar $=50 \mu \mathrm{m})$. 
In the LD hamster (Fig. 5B), JAM-A reactivity localised to the basal aspect of the seminiferous epithelium surrounding GCs residing on the basement membrane (open circle). JAM-A expression in the LD animal exhibited stage-specificity, with the highest levels occurring around stage VIII (data not shown). At stages where JAM-A was expressed, it co-localised strongly with basally located espin. Surprisingly, JAM-A reactivity was observed around GCs residing on the basement membrane in the SD animal (Fig. 5C, open circles). After 2 and 4 days of FSH treatment (Fig. 5D and E), JAM-A reactivity localised to the basal Sertoli cell aspect (open circle) and co-localisation with espin was low. By 10 days of FSH treatment (Fig. 5F), JAM-A expression was similar to that
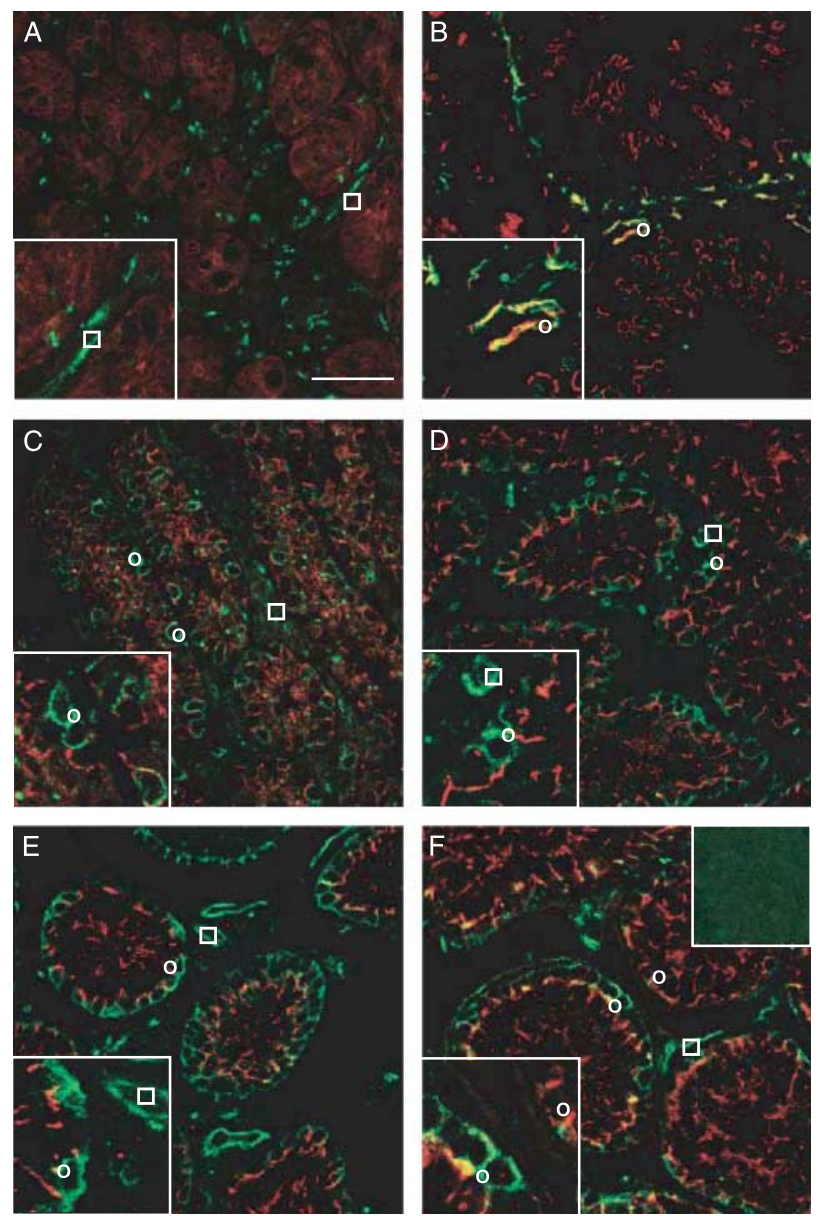

Figure 5 FSH regulates JAM-A localisation. The localisation of tight junction protein JAM-A (green, open circles) and the testis-specific adherens junction protein espin (red) by confocal immunofluorescence, in testes from the immature (day 5) hamster (A) and adult longday (LD) (B), short-day (SD) (C) and SD hamster treated with FSH for 2, 4 and 10 days ( $D, E$ and $F$ respectively). See the text for description of localisation. Lower inserts are enlarged regions of the same micrograph. The upper insert in $\mathrm{F}$ is a control where the primary antibody was substituted with an equivalent concentration of a nonspecific antibody from the same species. Open squares indicate JAM-A reactivity in blood vessels (bar $=50 \mu \mathrm{m})$. observed in the LD animal with an apparent stagedependency of localisation (open circles).

\section{Analysis of JAM-A expression in GCs}

The expression of JAM-A by GCs in the seminiferous epithelium has not been observed previously. To verify the expression of JAM-A by GCs, light microscopy was performed to identify cell types stained in the section (LD hamster; Fig. 6A). JAM-A localisation was observed at the Sertoli-Sertoli cell interface and also around basally located GCs (type A/intermediate spermatogonia, as well as preleptotene and leptotene spermatocytes (Fig. 6A, triangles and magnification of this image in left insert). JAM-A (F11r) mRNA was also present in high levels in murine GC1 and GC2 germ cell lines, and lower levels in TM3 (Leydig-like) and TM4 (Sertoli-like) lines (Fig. 6B). Finally, in silico analysis of JAM-A expression via the GermOnline website (www.germonline.org) for microarray data (Chalmel et al. 2007) identified high levels of JAM-A in purified rodent spermatogonia and Sertoli cells, but not spermatocytes or spermatids (data not shown).

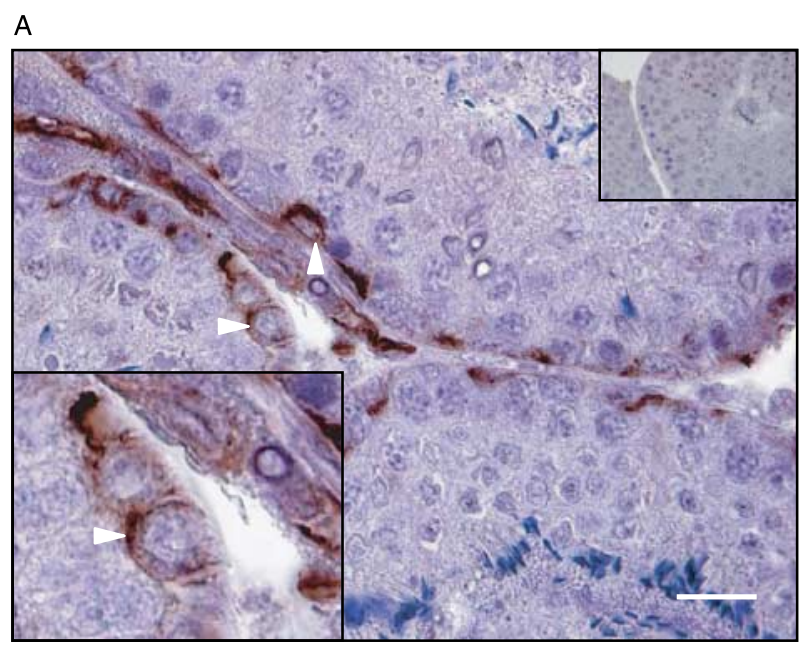

B STD GC1 GC2 TM3 TM4

JAM-A

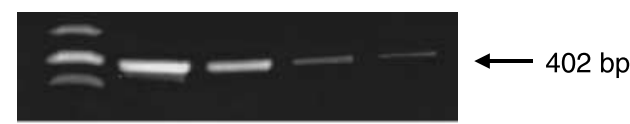

$\beta$-Actin

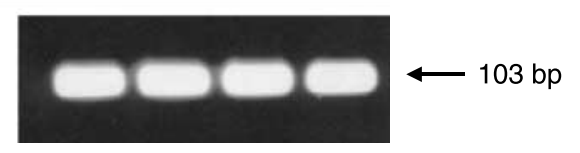

Figure 6 Analysis of JAM-A reactivity in germ cells. Light microscopic immunolocalisation of JAM-A protein in LD hamster (A, triangles). The right insert is an isotype control, while the left insert is an enlarged region highlighting JAM-A staining in germ cells at the basement membrane (bar $=50 \mu \mathrm{m})$. (B) PCR for JAM-A (F11r) in cDNA from mouse testicular germ cell (GC1 and GC2) and somatic cell lines (Leydig, TM3 and Sertoli, TM4). STD=standard ladder. 


\section{FSH regulates occludin localisation}

In the immature (day 5) hamster (Fig. 7A), a low-level punctate occludin reactivity was apparent in Sertoli cell cytoplasm (open circle), but this was not clearly distinguishable from the reactivity observed in the isotype control (data not shown). In the LD hamster (Fig. 7B), organised occludin reactivity localised in a scalloped pattern at the basal aspect of Sertoli cells and exhibited a stage-specific pattern of expression (data not shown). Where occludin was expressed, it co-localised strongly with espin (Fig. 7B, open circle). The SD hamster (Fig. 7C) exhibited intense but disorganised occludin reactivity that localised to the Sertoli cell cytoplasm (open circle). After 2 and 4 days of FSH treatment (Fig. 7D and $\mathrm{E}$ respectively), occludin reactivity co-localised with
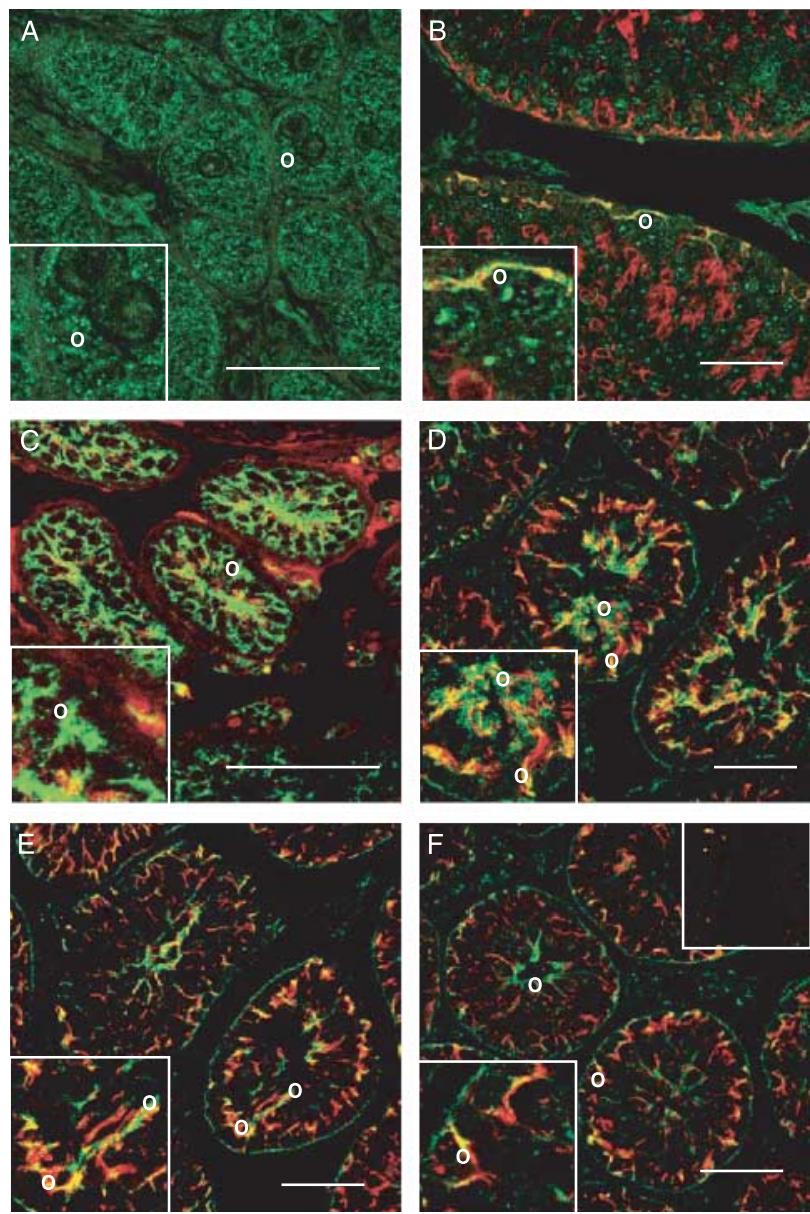

Figure 7 FSH regulates occludin localisation. The localisation of tight junction protein occludin (green, open circles) and the testis-specific adherens junction protein espin (red) by confocal immunofluorescence, in testes from the immature (day 5) hamster (A) and adult longday (LD) (B), short-day (SD) (C) and SD hamster treated with FSH for 2, 4 and 10 days (D, E and $F$ respectively). See the text for description of localisation. Lower inserts are enlarged regions of the same micrograph. The upper insert in $\mathrm{F}$ is a control where the primary antibody was substituted with an equivalent concentration of a nonspecific antibody from the same species (bar $=50 \mu \mathrm{m}$ ). espin along the basement membrane in an organised pattern similar to that seen in the LD animal, as well as in the apical regions of Sertoli cell cytoplasm (open circles). After 10 days of FSH treatment (Fig. 7F), a greater proportion of occludin reactivity localised to the basal aspect of Sertoli cells than in apical cytoplasm (open circles). Co-localisation with espin was observed at all FSH treatment time points.

\section{Gonadotrophin suppression increases tight junction mRNA but decreases ZO-1 mRNA expression}

The TJ proteins (claudin-11, claudin-3 and occludin; Fig. $8 \mathrm{~A}-\mathrm{C}$ respectively) all exhibited significant $(P<0.02)$ increases in mRNA expression (2.4-, 2.3- and threefold respectively) after gonadotrophin suppression. By contrast, mRNA levels for the adaptor protein ZO-1 (Fig. 8D) decreased (fourfold, $P<0.001$ ) after gonadotrophin suppression. The Sertoli cell-specific Gata6 was used as a housekeeper gene (Florin et al. 2005) to control for the reduction in Sertoli cell number known to occur in SD hamsters (Meachem et al. 2005). An additional, more commonly employed housekeeper ( $\beta$-actin) was employed with results similar to those presented here (data not shown). The quantitation of JAM-A (F11r) mRNA expression was not possible as we were unable to design primers that amplified hamster JAM-A, despite these working well for rat and mouse JAM-A analysis (not shown).

\section{Discussion}

This study has established that there is a clear stagedependency of tight junction protein localisation in the normal adult hamster testis. Via the use of claudin-11 as a protein marker of TJs, we have also shown that tight junction protein localisation correlates well with BTB function in the normal adult. The suppression of gonadotrophins resulted in the disorganisation of tight junction protein localisation and a loss of BTB function, which was reversible following FSH replacement. However, TJ mRNA expression was increased in gonadotrophin-suppressed animals. It is concluded that BTB function in the adult hamster testis is primarily regulated at the level of TJ protein organisation, but not at TJ mRNA expression.

The localisation of the TJ protein claudin-11 changes over the cycle of the seminiferous epithelium, from a predominantly basal phenotype at stages X-XII and I-III (relative to GCs residing on the basement membrane), adluminal at stages VII-VIII (relative to GCs residing on the basement membrane) and an intermediate profile at other stages. This pattern of organisation was closely associated with the BTB functionality as shown by the permeation of biotin tracer. The degree of claudin-11 reactivity appeared highest during stages VII-VIII, where preleptotene/leptotene spermatocytes are known to be preparing to traverse the BTB. Finally, our evidence 

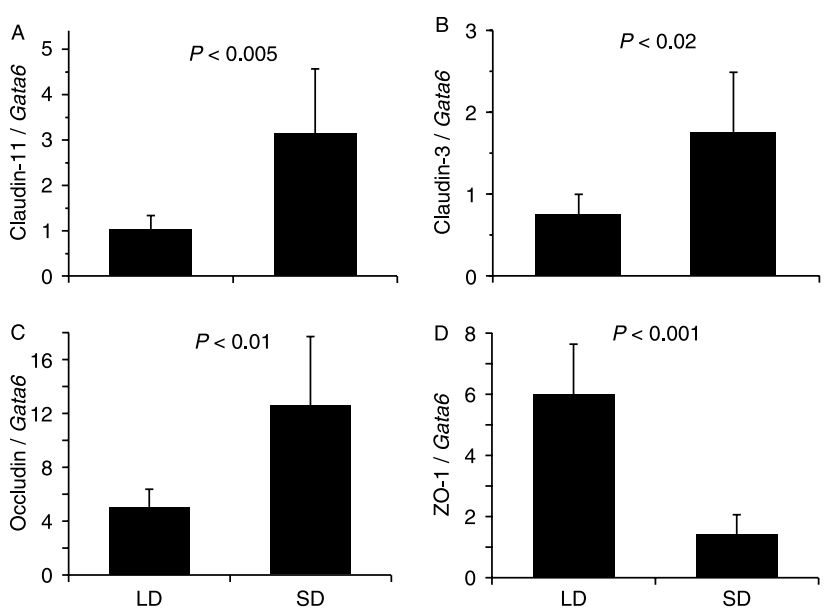

Figure 8 Gonadotrophin suppression increases tight junction mRNA and reduces adaptor protein mRNA. Quantitative real-time PCR analysis of tight junction proteins: claudin-11 (A), claudin-3 (B) and occludin (C) and adaptor protein ZO-1 (D) in the LD and SD hamster ( $n=$ seven to eight animals per group, results presented as mean \pm S.D).

indicates that JAM-A is found on both Sertoli and a subset of basal GCs in the testis, a finding that is supported by microarray data from Sertoli and GC isolates (Chalmel et al. 2007), as well as the identification of JAM-A protein in spermatozoa from the caudal epididymis (Shao et al. 2008). Together, these data suggest a novel role for JAM-A in spermatogenesis. In other tissues, JAM-A is involved in the movement of cells through epithelial and endothelial tissues, particularly leukocyte migration (for reviews see Bradfield et al. 2007). It is believed that this action is achieved by the interactions of JAM-A with various intracellular binding partners (such as partitioning defective proteins PAR3/ PAR6 and RAP-1) to affect cytoskeletal reorganisation (for reviews see Bos 2005, Mandell et al. 2005). The movement of pre-meiotic GCs across BTB involves significant cytoskeletal reorganisation, and the identification of JAM-A expression in these GCs leads us to speculate that this tight junction protein may serve as a homophilic partner for Sertoli cell JAM-A, to facilitate GC migration through the BTB. JAM-A expression is then lost in most GCs resident in the adluminal compartment. A similar localisation and involvement in GC migration has recently been hypothesised for a transmembrane protein similar to JAM-A; coxsackie and adenovirus receptor (reviewed in Wang \& Cheng 2007, Wang et al. 2007).

Evidence indicates that androgens up-regulate claudin-3 mRNA and protein (Meng et al. 2005), and claudin-11 mRNA (Florin et al. 2005) and protein localisation in the rodent (Kaitu'u-Lino et al. 2007). Thus, we hypothesised that the natural suppression of gonadotrophins in the SD Djungarian hamster would result in decreased tight junction mRNA levels, protein organisation and BTB functionality. Our hypothesis is supported in terms of protein localisation and function by the data presented here, but not for mRNA levels for claudin-3, claudin-11 and occludin, all of which increased following gonadotrophin suppression. By contrast, mRNA levels for the intracellular adaptor protein ZO-1 decreased after gonadotrophin suppression. ZO-1 is presumed to be responsible for assembly, scaffolding and regulation of transmembrane TJ proteins (Anderson et al. 1995, Denker \& Nigam 1998), through its ability to bind both transmembrane TJ proteins and the underlying actin cytoskeleton (Fanning et al. 2002). The dissociation of ZO-1 from transmembrane TJ has also been shown to be associated with barrier regulation in the gut (Pappenheimer 1987, Kawkitinarong et al. 2004), and a similar mechanism of barrier regulation may be occurring in the testis. Whereas androgens have been shown to regulate $\mathrm{TJ}$ proteins in mouse and rat models (Florin et al. 2005, Meng et al. 2005, Kaitu'u-Lino et al. 2007), our data reported here demonstrate that FSH is also able to regulate TJ protein localisation and function in the hamster, and thus it appears that there is a differential response to gonadotrophins at testicular TJs across species.

The decrease in organisation and the increase in tight junction mRNA observed in the SD hamster were unexpected and prompt a re-evaluation of our understanding of $\mathrm{TJ}$ regulation. The absence of a functional BTB in the SD hamster is established from earlier studies (Bergmann 1987) and confirmed by the data presented here. The results from the present study strongly indicate that organisation of TJ protein and not TJ protein expression itself is central to barrier functionality. Thus, following other epithelial tissue systems (Hopkins et al. 2003, Morimoto et al. 2005), testicular tight junction proteins may be recycled into disorganised intracellular pools after gonadotrophin suppression in this model. Upon gonadotrophin stimulation, it is proposed that tight junction proteins would relocate to the cell membrane, providing a mechanism conducive to a rapid reorganisation of functional tight junctions and re-initiation of spermatogenesis. The recycling of membrane-bound versus intracellular TJ proteins has recently been implicated in TJ regulation elsewhere (Sarkar et al. 2008, Xia et al. 2007). Our data demonstrates that such testicular tight junction protein recycling after gonadotrophin suppression occurs for claudin-11, occludin and Sertoli cell JAM-A, but not claudin-3 or GC JAM-A.

In conclusion, gonadotrophin suppression in the Djungarian hamster disrupts testicular TJ organisation and increases TJ mRNA levels, but decreases mRNA levels for the adaptor protein ZO-1. FSH replacement induces a rapid reorganisation of $\mathrm{TJ}$ protein within 2 days. Reactivity and local isation of the major TJ protein at the BTB, claudin11 , changed over the cycle of the seminiferous epithelium, with tubules at stages I-III and XI-XII exhibiting a very lowlevel reactivity and expression largely isolated to the basement membrane. At stages IV-VI and IX-X, claudin-11 
expression and localisation were intermediate, and adluminal at stages VII-VIII. The extent of biotin tracer permeation was strongly associated with the localisation of claudin-11 expression. Taken together, this study provides clear evidence that the organisation and localisation, rather than expression, of TJ proteins determine BTB functionality in the testis.

\section{Materials and Methods}

\section{Animals}

Djungarian hamster (Phodopus sungorus) testicular tissues were obtained from archived material collected at the University of Münster, Germany (for details see Meachem et al. (2005) and Tarulli et al. (2006)). For the assessment of claudin-11 protein localisation and BTB functionality, 15 pubertal $(5 \times \mathrm{d} 5,5 \times \mathrm{d} 15$ and $5 \times \mathrm{d} 30), 5$ adult LD and 15 adult SD hamsters were bred, raised and housed for $90 \pm 30$ days (for adult animals) in the colony of the Department of Cell Biology and Physiology, University of Pittsburgh, Pennsylvania. All experiments were in accordance with local guidelines and laws on the care and use of laboratory animals.

\section{Experimental design}

Gonadotrophin suppression and testicular function were suppressed by transfer of adult hamsters $(150 \pm 30$ days, $n=$ five to seven per group) from long-day (LD, $16 \mathrm{~h}$ light: $8 \mathrm{~h}$ darkness) to SD photoperiod exposure (SD, $8 \mathrm{~h}$ light: $16 \mathrm{~h}$ darkness) for 12 weeks. One group of hamsters remained under long photoperiods as reproductively active controls. The response to photoinhibition was assessed by palpation after which all hamsters with no palpable testes were included in the study. Hamsters received FSH treatment for 2, 4 and 10 days, where animals received $6 \mathrm{IU} /$ day of either recombinant human FSH (Gonal-F Serono; Meachem et al. 2005) or highly purified human urinary FSH (Metrodin-HP, Serono), reconstituted in sterile $0.154 \mathrm{M} \mathrm{NaCl}$. This dose significantly increased serum FSH in this model, with no change in serum or testicular testosterone (Meachem et al. 2005). Metrodin-HP has been shown elsewhere to have negligible LH activity (<0.002\%; Howles 1996, Revelli et al. 2006). One group of hamsters remained under SD conditions and received no treatment, serving as reproductively inactive SD controls. The testes were excised, weighed, immersion-fixed in Bouin's solution $(5 \mathrm{~h})$ and embedded in paraffin (Tarulli et al. 2006), or immediately frozen for mRNA extraction (Meachem et al. 2005).

\section{Immunohistology}

Sections $(5 \mu \mathrm{m})$ were dewaxed in histolene $(2 \times 8 \mathrm{~min})$ and $100 \%$ ethanol ( $5 \mathrm{~min}$ ) before air drying and rehydration in graded ethanol (90,75 and 50\%) and finally in deionised water. Antigen retrieval was then performed by immersing sections in $600 \mathrm{ml}$ of $1 \mathrm{mMEDTA}-\mathrm{NaOH}(\mathrm{pH} 8.0)$ and heating in an $800 \mathrm{~W}$ microwave at $100 \%$ power for $5 \mathrm{~min}$, standing at room temperature for $5 \mathrm{~min}$, then low simmer (20\% power) for an additional $5 \mathrm{~min}$, before cooling for $1 \mathrm{~h}$ in EDTA buffer. The sections were then washed in deionised water. Primary antibodies were then applied as outlined in Table 1, in $10 \mathrm{mM}$ PBS with $10 \%$ normal serum from the species in which the secondary antibody was raised (normal goat serum (Chemicon International, Temecula, CA, USA)). A primary antibody to espin, a component of the ectoplasmic specialisation (a testisspecific adherens junction) known to be present at inter-Sertoli cell and Sertoli-GC junctions, was employed in conjunction with antibodies to TJ in the assessment of BTB organisation. It has been established that TJ and adherens junctions coexist at the BTB (Yan \& Cheng 2005). This provided a constant marker (espin, found at the region of the BTB at all stages) to compare with the organisation of TJ proteins found only at specific stages of the seminiferous epithelial cycle. The detection of primary antibody, verification of specificity and mounting of tissue were performed as described elsewhere (Tarulli et al. 2006). The sections were visualised on a confocal microscope (Fluoview FV300, Olympus Australia, Mt Waverley, Australia).

\section{$R T$ and real-time PCR analysis}

Real-time PCR was used to measure the relative mRNA levels of TJ and associated proteins in LD and SD control animals (Meachem et al. 2005). Total RNA was isolated using an RNA extraction column (Qiagen) and then treated to remove residual genomic DNA (Ambion DNA-free treatment kit; Ambion, Austin TX, USA). RNA (1 ug) was reverse-transcribed using Superscript II in a final volume of $20 \mu \mathrm{l}$ according to the manufacturer's protocol (Invitrogen). For each sample, the absence of contaminating genomic DNA in cDNA samples was confirmed using reactions in which the RT enzyme was omitted. Quantitative RT-PCR analysis was performed using the Roche LightCycler (Roche) and the FastStart DNA Master SYBR-green 1 system (Roche Cat\# 12239264001). Oligonucleotide primers for claudin-11, claudin-3, occludin, ZO-1 (Tjp1), Gata6 and $\beta$-actin were designed using Oligo6 (Molecular Biology Insights, Cascade, CO, USA) based on the corresponding mouse sequence obtained from the PubMed nucleotide database (Table 2). For PCR analysis,

Table 1 Primary antibody details.

\begin{tabular}{|c|c|c|c|c|c|c|}
\hline Antigen & Marker & Supplier & Cat. No. & Host & Concentration (ug/ml) & Incubation time \\
\hline Claudin-3 & Tight junctions & Zymed Labs ${ }^{\mathrm{a}}$ & $34-1700$ & Rabbit & 2 & $2 \mathrm{~h}$ \\
\hline Occludin & Tight junctions & Zymed Labs & $71-1500$ & Rabbit & 2 & $2 \mathrm{~h}$ \\
\hline JAM-A & Tight junctions & Zymed Labs & $36-1700$ & Rabbit & 1 & $1 \mathrm{~h}$ \\
\hline Claudin-11 & Tight junctions & Zymed Labs & $36-4500$ & Rabbit & 1 & $2 \mathrm{~h}$ \\
\hline Espin & $\begin{array}{l}\text { Testis-specific adherens } \\
\text { junctions }\end{array}$ & $\begin{array}{l}\text { BD Transduction } \\
\text { Labs }^{b}\end{array}$ & 611656 & Mouse & 0.75 & $30 \mathrm{~min}$ \\
\hline
\end{tabular}

${ }^{a}$ Zymed Laboratories Inc., South San Francisco, CA, USA. ${ }^{b}$ BD Transduction Laboratories, Franklin Lakes, NJ, USA. 
sample cDNA was diluted 1:50- to 1:400-fold, and PCR conditions, including $\mathrm{Mg}^{2+}$ concentration, primer concentration, annealing time and extension time were optimised for each primer pair as summarised in Table 2. For all PCR analyses, standard curves were generated using dilutions of pooled LD hamster testicular cDNA preparation assigned an arbitrary unitage. All samples were analysed in triplicate for $\sim 35-40$ cycles after which a melting curve analysis was performed to monitor PCR product purity. All PCR product identities were confirmed by DNA sequencing (data not shown). The results were expressed in terms of the housekeeper gene Gata6, known to be expressed in the testis exclusively by the Sertoli cells (Florin et al. 2005). Experiments were repeated using an additional housekeeper $\beta$-actin $(A c t b)$, with no change in results (data not shown).

\section{Real-time PCR analysis of JAM-A (F11r) mRNA in germ and somatic cell lines}

mRNA was extracted and cDNA prepared, as outlined above, from murine GC1 and GC2 (pre-meiotic GC lines; Hofmann et al. 1992, Wolkowicz et al. 1996), TM3 (Leydig-like) and TM4 (Sertoli-like; Mather 1980) cell lines grown to confluence, as described elsewhere (Farnworth et al. 2007). PCR conditions were as outlined in Table 2.

\section{Light microscopic analysis of JAM-A expression in GCS}

After primary antibody incubation (see above), the sections were incubated with biotinylated secondary goat anti-rabbit polyclonal antibody for $30 \mathrm{~min}$. After washing in PBS $(2 \times 5 \mathrm{~min})$, the sections were incubated with Vector $A B C$ reagent (Vector Labs, Burlingame, CA, USA; Cat. No. PK-6100) for $15 \mathrm{~min}$. The sections were resolved employing 3,3'-diaminobenzidine chromogen (Dako, Carpinteria, CA, USA; Cat. No. K3466) and mounted under an aqueous solution (see above).

\section{Assessment of stage-dependency of tight junction localisation and BTB functionality over the cycle of the seminiferous epithelium}

All testes were injected with NHS-linked biotin (Pierce Scientific, Rockford, IL, USA; Cat. No. 21336) immediately after excision (Meng et al. 2005). Biotin was allowed to perfuse for $30 \mathrm{~min}$ before testes were fixed and embedded as outlined above. The sections were cut and stained for claudin-11 (see above), with detection by anti-rabbit-Alexa 647 (for claudin11), PNA-lectin-Alexa 546 (to allow for tubule staging) and streptavidin-Alexa-488 (to allow the visualisation of qualitative biotin tracer; Invitrogen).

\section{Quantitation of stage-dependent tight junction expression}

Confocal immunohistology was performed in LD hamsters, as described above employing the claudin-11 antibody, to quantify stage-dependent TJ expression patterns. Localisation of claudin-11 was classified as basal, intermediate or apical relative to GCs residing on the basement membrane (these include www.reproduction-online.org

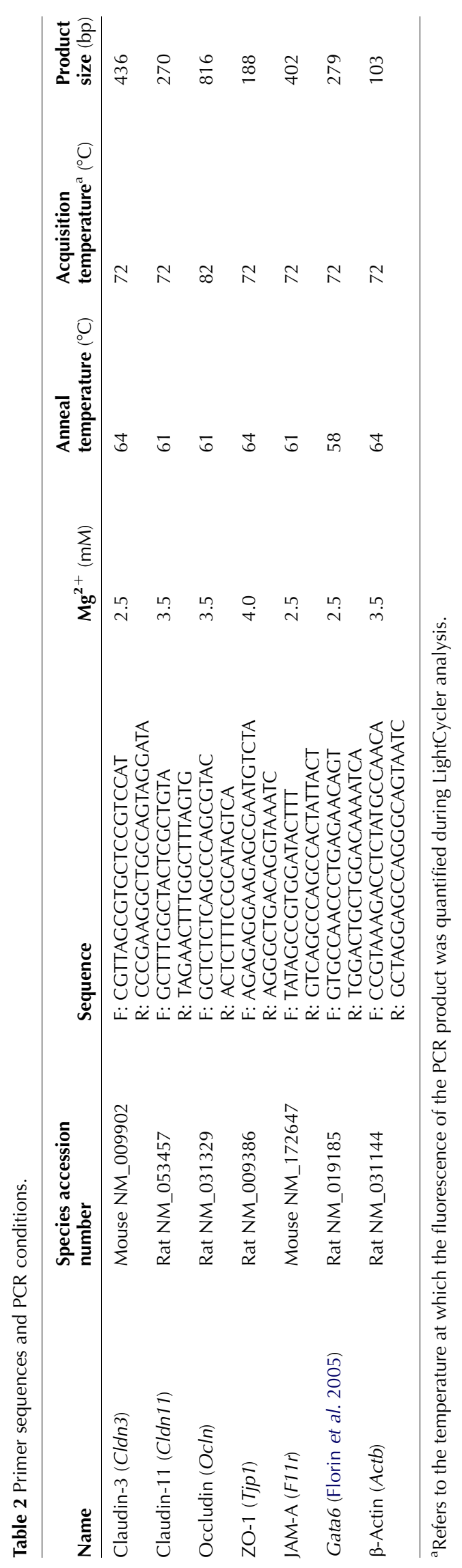

Reproduction (2008) 135 867-877 
spermatogonia through to preleptotene spermatocytes). Fields were selected using a systematic uniform approach from a random start and images collected. An unbiased counting frame (Tarulli et al. 2006) was superimposed on each image, evaluated and counted whether a section of the entire tubular epithelium fell within the frame or touched the acceptance boundary. The examples of each grouping are illustrated in Fig. 1A. Tubules were staged in the following groups: stages I-III, stages IV-VI, stages VII-VIII, stages IX-X and stages XI-XII. A minimum of 20 tubules were counted per stage grouping, per animal, for five animals. The extent of claudin- 11 reactivity across the tubule was defined qualitatively with the following scale $(-,+,++,+++)$, where $(-)$ and $(+++)$ reflect no reactivity and maximal reactivity respectively, and $(+),(++)$ are intermediary levels.

\section{Statistical analysis}

For mRNA analysis, the samples were run in triplicate and data were assessed for homogeneity of variance after which a twosample $t$-test was used to compare mRNA levels in LD and SD control samples, with differences $P<0.05$ considered significant. Each experiment was performed twice, and representative data are shown.

\section{Acknowledgements}

We gratefully acknowledge Mark McCabe from Prince Henry's Institute for his assistance with the biotin tracer technique, Scott Hergenrother from the University of Pittsburgh for his assistance with animal experimentation, and Yao Wang and Paul Farnworth for their provision of GC1, GC2, TM3 and TM4 cDNA. Supported by the National Health and Medical Research Council of Australia Program Grant \#241000 (S J M, P G S) and National Institute of Health Grant: 2U54 HD008610 (S S). The authors declare that there is no conflict of interest that would prejudice the impartiality of this scientific work.

\section{References}

Anderson JM, Fanning AS, Lapierre L \& Van Itallie CM 1995 Zonula occludens (ZO)-1 and ZO-2: membrane-associated guanylate kinase homologues (MAGuKs) of the tight junction. Biochemical Society Transactions 23 470-475.

Bartles JR, Wierda A \& Zheng L 1996 Identification and characterization of espin, an actin-binding protein localized to the F-actin-rich junctional plaques of Sertoli cell ectoplasmic specializations. Journal of Cell Science 109 1229-1239.

Bazzoni G, Martinez-Estrada OM, Orsenigo F, Cordenonsi M, Citi S \& Dejana E 2000 Interaction of junctional adhesion molecule with the tight junction components ZO-1, cingulin, and occludin. Journal of Biological Chemistry 275 20520-20526.

Bergmann M 1987 Photoperiod and testicular function in Phodopus sungorus. Advances in Anatomy, Embryology and Cell Biology 105 1-76.

Bos JL 2005 Linking Rap to cell adhesion. Current Opinion in Cell Biology 17 123-128.

Bradfield PF, Nourshargh S, Aurrand-Lions M \& Imhof BA 2007 JAM family and related proteins in leukocyte migration (Vestweber series). Arteriosclerosis, Thrombosis and Vascular Biology 27 2104-2112.

Cavicchia JC \& Sacerdote FL 1991 Correlation between blood-testis barrier development and onset of the first spermatogenic wave in normal and in busulfan-treated rats: a lanthanum and freeze-fracture study. Anatomical Record 230 361-368.
Chalmel F, Rolland AD, Niederhauser-Wiederkehr C, Chung SS, Demougin P, Gattiker A, Moore J, Patard JJ, Wolgemuth DJ, Jegou B et al. 2007 The conserved transcriptome in human and rodent male gametogenesis. PNAS $1048346-8351$.

Denker BM \& Nigam SK 1998 Molecular structure and assembly of the tight junction. American Journal of Physiology 274 F1-F9.

Fanning AS, Jameson BJ, Jesaitis LA \& Anderson JM 1998 The tight junction protein ZO-1 establishes a link between the transmembrane protein occludin and the actin cytoskeleton. Journal of Biological Chemistry 273 29745-29753.

Fanning AS, Ma TY \& Anderson JM 2002 Isolation and functional characterization of the actin binding region in the tight junction protein ZO-1. FASEB Journal 16 1835-1837.

Farnworth PG, Wang Y, Escalona R, Leembruggen P, Ooi GT \& Findlay JK 2007 Transforming growth factor-beta blocks inhibin binding to different target cell types in a context-dependent manner through dual mechanisms involving betaglycan. Endocrinology 148 5355-5368.

Florin A, Maire M, Bozec A, Hellani A, Chater S, Bars R, Chuzel F \& Benahmed M 2005 Androgens and postmeiotic germ cells regulate claudin-11 expression in rat Sertoli cells. Endocrinology 146 1532-1540.

Furuse M, Hirase T, Itoh M, Nagafuchi A, Yonemura S \& Tsukita S 1993 Occludin: a novel integral membrane protein localizing at tight junctions. Journal of Cell Biology 123 1777-1788.

Furuta I, Porkka-Heiskanen T, Scarbrough K, Tapanainen J, Turek FW \& Hsueh AJ 1994 Photoperiod regulates testis cell apoptosis in Djungarian hamsters. Biology of Reproduction 51 1315-1321.

Gow A, Southwood CM, Li JS, Pariali M, Riordan GP, Brodie SE, Danias J, Bronstein JM, Kachar B \& Lazzarini RA 1999 CNS myelin and sertoli cell tight junction strands are absent in Osp/claudin-11 null mice. Cell 99 649-659.

Grima J, Wong CC, Zhu LJ, Zong SD \& Cheng CY 1998 Testin secreted by Sertoli cells is associated with the cell surface, and its expression correlates with the disruption of Sertoli-germ cell junctions but not the inter-Sertoli tight junction. Journal of Biological Chemistry 273 21040-21053.

Hellani A, Ji J, Mauduit C, Deschildre C, Tabone E \& Benahmed M 2000 Developmental and hormonal regulation of the expression of oligodendrocyte-specific protein/claudin 11 in mouse testis. Endocrinology 141 3012-3019.

Hofmann MC, Narisawa S, Hess RA \& Millan JL 1992 Immortalization of germ cells and somatic testicular cells using the SV40 large T antigen. Experimental Cell Research 201 417-435.

Hopkins AM, Walsh SV, Verkade P, Boquet P \& Nusrat A 2003 Constitutive activation of Rho proteins by CNF-1 influences tight junction structure and epithelial barrier function. Journal of Cell Science 116 725-742.

Howles CM 1996 Genetic engineering of human FSH (Gonal-F ${ }^{\circledR}$ ). Human Reproduction Update 2 172-191.

Janecki A, Jakubowiak A \& Steinberger A 1991 Effects of cyclic AMP and phorbol ester on transepithelial electrical resistance of Sertoli cell monolayers in two-compartment culture. Molecular and Cellular Endocrinology 82 61-69.

Kaitu'u-Lino TJ, Sluka P, Foo CF \& Stanton PG 2007 Claudin-11 expression and localisation is regulated by androgens in rat Sertoli cells in vitro. Reproduction 133 1169-1179.

Kawkitinarong K, Linz-McGillem L, Birukov KG \& Garcia JG 2004 Differential regulation of human lung epithelial and endothelial barrier function by thrombin. American Journal of Respiratory Cell and Molecular Biology 31 517-527.

Lee NP \& Cheng CY 2004 Adaptors, junction dynamics, and spermatogenesis. Biology of Reproduction 71 392-404.

Lerchl A, Sotiriadou S, Behre HM, Pierce J, Weinbauer GF, Kliesch S \& Nieschlag E 1993 Restoration of spermatogenesis by follicle-stimulating hormone despite low intratesticular testosterone in photoinhibited hypogonadotropic Djungarian hamsters (Phodopus sungorus). Biology of Reproduction 49 1108-1116.

Li JC, Mruk D \& Cheng CY 2001 The inter-Sertoli tight junction permeability barrier is regulated by the interplay of protein phosphatases and kinases: an in vitro study. Journal of Andrology 22 847-856.

Li MW, Xia W, Mruk DD, Wang CQ, Yan HH, Siu MK, Lui WY, Lee WM \& Cheng CY 2006 Tumor necrosis factor $\alpha$ reversibly disrupts the bloodtestis barrier and impairs Sertoli-germ cell adhesion in the seminiferous epithelium of adult rat testes. Journal of Endocrinology 190 313-329. 
Lui WY, Lee WM \& Cheng CY 2001 Transforming growth factor-beta3 perturbs the inter-Sertoli tight junction permeability barrier in vitro possibly mediated via its effects on occludin, zonula occludens-1, and claudin-11. Endocrinology 142 1865-1877.

Lui WY, Mruk D, Lee WM \& Cheng CY 2003 Sertoli cell tight junction dynamics: their regulation during spermatogenesis. Biology of Reproduction 68 1087-1097.

Mandell KJ, Babbin BA, Nusrat A \& Parkos CA 2005 Junctional adhesion molecule 1 regulates epithelial cell morphology through effects on beta1 integrins and Rap1 activity. Journal of Biological Chemistry 280 11665-11674.

Martin-Padura I, Lostaglio S, Schneemann M, Williams L, Romano $M$, Fruscella P, Panzeri C, Stoppacciaro A, Ruco L, Villa A et al. 1998 Junctional adhesion molecule, a novel member of the immunoglobulin superfamily that distributes at intercellular junctions and modulates monocyte transmigration. Journal of Cell Biology 142 117-127.

Mather JP 1980 Establishment and characterization of two distinct mouse testicular epithelial cell lines. Biology of Reproduction 23 243-252.

Matter K \& Balda MS 2007 Epithelial tight junctions, gene expression and nucleo-junctional interplay. Journal of Cell Science 120 1505-1511.

Meachem SJ, Stanton PG \& Schlatt S 2005 Follicle-stimulating hormone regulates both Sertoli cell and spermatogonial populations in the adult photoinhibited Djungarian hamster testis. Biology of Reproduction 72 $1187-1193$.

Meachem SJ, Schlatt S, Ruwanpura SM \& Stanton PG 2007 The effect of testosterone, dihydrotestosterone and oestradiol on the re-initiation of spermatogenesis in the adult photoinhibited Djungarian hamster. Journal of Endocrinology 192 553-561.

Meng J, Holdcraft RW, Shima JE, Griswold MD \& Braun RE 2005 Androgens regulate the permeability of the blood-testis barrier. PNAS 102 16696-16700.

Morales A, Mohamed F \& Cavicchia JC 2007 Apoptosis and blood-testis barrier during the first spermatogenic wave in the pubertal rat. Anatomical Record 290 206-214.

Morimoto S, Nishimura N, Terai T, Manabe S, Yamamoto Y, Shinahara W, Miyake H, Tashiro S, Shimada M \& Sasaki T 2005 Rab13 mediates the continuous endocytic recycling of occludin to the cell surface. Journal of Biological Chemistry 280 2220-2228.

Morita K, Sasaki H, Fujimoto K, Furuse M \& Tsukita S 1999 Claudin11/OSP-based tight junctions of myelin sheaths in brain and Sertoli cells in testis. Journal of Cell Biology 145 579-588.

Morris AP, Tawil A, Berkova Z, Wible L, Smith CW \& Cunningham SA 2006 Junctional adhesion molecules (JAMs) are differentially expressed in fibroblasts and co-localize with ZO-1 to adherens-like junctions. Cell Communication \& Adhesion 13 233-247.

Niklowitz P, Lerchl A \& Nieschlag E 1997 In vitro fertilizing capacity of sperm from FSH-treated photoinhibited Djungarian hamsters (Phodopus sungorus). Journal of Endocrinology 154 475-481.

Pappenheimer JR 1987 Physiological regulation of transepithelial impedance in the intestinal mucosa of rats and hamsters. Journal of Membrane Biology 100 137-148.

Rehder D, Iden S, Nasdala I, Wegener J, Brickwedde MK, Vestweber D \& Ebnet K 2006 Junctional adhesion molecule - a participates in the formation of apico-basal polarity through different domains. Experimental Cell Research 312 3389-3403.

Revelli A, Poso F, Gennarelli G, Moffa F, Grassi G \& Massobrio M 2006 Recombinant versus highly-purified, urinary follicle-stimulating hormone (r-FSH vs. HP-uFSH) in ovulation induction: a prospective, randomized study with cost-minimization analysis. Reproductive Biology and Endocrinology 4 38-44.

Sarkar O, Mathur PP, Cheng CY \& Mruk DD 2008 Interleukin 1 Alpha (IL1A) is a novel regulator of the blood-testis barrier in the rat. Biology of Reproduction 75 445-454.
Schlatt S, DeGeyter M, Kliesch S, Nieschlag E \& Bergmann M 1995 Spontaneous recrudescence of spermatogenesis in the photoinhibited male Djungarian hamster, Phodopus sungorus. Biology of Reproduction 53 1169-1177.

Setchell BP, Pollanen P \& Zupp JL 1988 Development of the blood-testis barrier and changes in vascular permeability at puberty in rats. International Journal of Andrology 11 225-233.

Setchell BP, Hertel T \& Soder O 2003 Postnatal testicular development, cellular organization and paracrine regulation. Endocrine Development 5 24-37.

Shao M, Ghosh A, Cooke VG, Naik UP \& Martin-DeLeon PA 2008 JAM-A is present in mammalian spermatozoa where it is essential for normal motility. Developmental Biology 313 246-255.

Stevenson BR, Siliciano JD, Mooseker MS \& Goodenough DA 1986 Identification of ZO-1: a high molecular weight polypeptide associated with the tight junction (zonula occludens) in a variety of epithelia. Journal of Cell Biology 103 755-766.

Tan KA, De Gendt K, Atanassova N, Walker M, Sharpe RM, Saunders PT, Denolet E \& Verhoeven G 2005 The role of androgens in Sertoli cell proliferation and functional maturation: studies in mice with total or Sertoli cell-selective ablation of the androgen receptor. Endocrinology 146 2674-2683.

Tarulli GA, Stanton PG, Lerchl A \& Meachem SJ 2006 Adult sertoli cells are not terminally differentiated in the Djungarian hamster: effect of FSH on proliferation and junction protein organization. Biology of Reproduction 74 798-806.

Turner JR 2006 Molecular basis of epithelial barrier regulation: from basic mechanisms to clinical application. American Journal of Pathology 169 1901-1909.

Wang CQ \& Cheng CY 2007 A seamless trespass: germ cell migration across the seminiferous epithelium during spermatogenesis. Journal of Cell Biology 178 549-556.

Wang CQ, Mruk DD, Lee WM \& Cheng CY 2007 Coxsackie and adenovirus receptor (CAR) is a product of Sertoli and germ cells in rat testes which is localized at the Sertoli-Sertoli and Sertoli-germ cell interface. Experimental Cell Research 313 1373-1392.

Wolkowicz MJ, Coonrod SA, Reddi PP, Millan JL, Hofmann MC \& Herr JC 1996 Refinement of the differentiated phenotype of the spermatogenic cell line GC-2spd(ts). Biology of Reproduction 55 923-932.

Woodfin A, Reichel CA, Khandoga A, Corada M, Voisin MB, Scheiermann C, Haskard DO, Dejana E, Krombach F \& Nourshargh S 2007 JAM-A mediates neutrophil transmigration in a stimulus-specific manner in vivo: evidence for sequential roles for JAM-A and PECAM-1 in neutrophil transmigration. Blood $1101848-1856$.

Xia W, Wong CH, Lee NP, Lee WM \& Cheng CY 2005 Disruption of Sertoligerm cell adhesion function in the seminiferous epithelium of the rat testis can be limited to adherens junctions without affecting the bloodtestis barrier integrity: an in vivo study using an androgen suppression model. Journal of Cell Physiology 205 141-157.

Xia W, Mruk DD \& Cheng CY 2007 C-type natriuretic peptide regulates blood-testis barrier dynamics in adult rat testes. PNAS 104 3841-3846.

Yan HH \& Cheng CY 2005 Blood-testis barrier dynamics are regulated by an engagement/disengagement mechanism between tight and adherens junctions via peripheral adaptors. PNAS 102 11722-11727.

Zen K, Liu Y, McCall IC, Wu T, Lee W, Babbin BA, Nusrat A \& Parkos CA 2005 Neutrophil migration across tight junctions is mediated by adhesive interactions between epithelial coxsackie and adenovirus receptor and a junctional adhesion molecule-like protein on neutrophils. Molecular and Cellular Biology 16 2694-2703.

Received 20 December 2007

First decision 28 January 2008

Accepted 5 March 2008 